\title{
Cardiac myosin activator shows promise in phase II trial
}

A novel, small-molecule, sarcomeredirected activator of cardiac myosin, omecamtiv mecarbil, is well tolerated in healthy individuals, and significantly improves cardiac function in patients with stable heart failure (HF), according to two reports published in The Lancet. Professor John Cleland, from the University of Hull, UK, is "excited about the possibility of a new molecule that could improve cardiac function not only by a fundamentally different molecular mechanism, but also a very different physiological principle (prolongation of contraction rather than increased velocity of contraction)."

Dr Fady Malik "initiated this research ... based on the observation that motor proteins, of which myosin is one, could be activated by small molecules." During cardiac contraction, myosin first weakly and then strongly binds actin to initiate cross-bridge cycling and force generation. By contrast with existing inotropes, omecamtiv mecarbil improves myocardial contraction by enhancing the transition rate between the bound states, and by prolonging the duration of systolic ejection without changing the rate of contraction.

In a first-in-man, dose-escalating study, 34 healthy men were given a $6 \mathrm{~h}$ intravenous infusion of omecamtiv mecarbil or placebo once per week for 4 weeks. The maximum tolerated dose of the active drug was $0.5 \mathrm{mg} / \mathrm{kg} / \mathrm{h}$. "Omecamtiv mecarbil increases the duration of systole in a highly predictable, dose-related fashion, resulting in increases in stroke volume and ejection fraction, without evidence of increased myocardial oxygen demand," states Professor John Teerlink, who has worked on the development of the drug since 2000.

Next, in a randomized, controlled, doseranging, phase II trial involving 45 patients with stable systolic HF (ejection fraction $\leq 40 \%$ ), omecamtiv mecarbil resulted in concentration-dependent increases in left ventricular ejection time and stroke volume, with a small reduction in heart rate. High plasma concentrations of the drug $(>500 \mathrm{ng} / \mathrm{ml})$ were associated with reductions in end-systolic and end-diastolic volumes, but also the emergence of cardiac ischemia in two patients.

"A new agent, such as omecamtiv mecarbil, that improves cardiac function without increasing energy expenditure and is not arrhythmogenic could be invaluable as an additional treatment for patients who have had an inadequate response to existing [inotropic] agents," says Professor Cleland. The researchers are investigating the oral formulation of the drug, and Professor Teerlink is chair of a phase IIb trial (ATOMIC-AHF), in which use of the intravenous agent in patients with acute $\mathrm{HF}$ will be tested.

Gregory B. Lim

Original articles Teerlink, J. R. et al. Dose-dependent augmentation of cardiac systolic function with the selective cardiac myosin activator, omecamtiv mecarbil: a first-in-man study. Lancet 378, 667-675 (2011) | Cleland, J. G. et al. The effects of the cardiac myosin activator, omecamtiv mecarbil, on cardiac function in systolic heart failure: a double-blind, placebo-controlled, crossover, dose-ranging phase 2 trial. Lancet 378, 676-683 (2011) 